\title{
Structural basis for the changing physical properties of human pulmonary vessels with age
}

\author{
E. H. MACKAY' , J. BANKS ${ }^{2}$, B. SYKES ${ }^{1}$, AND G. DE J. LEE $^{2}$ \\ From the Departments of Pathology ${ }^{1}$, and Cardiology ${ }^{2}$, Radcliffe Infirmary, Oxford
}

\begin{abstract}
MacKay, E. H., Banks, J., Sykes, B., and Lee, G. de J. (1978). Thorax, 33, 335-344. Structural basis for the changing physical properties of human pulmonary vessels with age. Circumferential strips of pulmonary vessel wall were obtained at necropsy from the major arterial and venous branches at the lung hilum in patients aged 7-87 years.

The extensibility of these strips was measured using the tension balance method of Harris et al. (British Heart Journal, 1965, 27, 651-659). The vessels were then bisected, and half of each strip was submitted for structural analysis using morphometric methods on paraffin sections stained to show the collagen, elastin, and muscle content. The other halves of the formalin-fixed vessel strips were examined chemically to determine their collagen content by estimation of the total hydroxyproline content. The thickness of the vessel media was measured microscopically on all of the sections examined.

Quantitative measurements were made on 42 arteries and 37 veins. Contrary to expectation, there was a steady fall in medial collagen content with increasing age in arteries and veins. The decrease in collagen content was similar in the morphometric and chemical studies and was statistically significant. The thickness of the vessel media did not change significantly with age.

The pulmonary artery and vein strips were less extensible in the older age groups, the main change occurring in the elastic phase of the vascular stress/strain curves. It is suggested that changes in the elastic tissue at a molecular and lamellar level are responsible for the increasing stiffness of pulmonary vessels rather than changes in the medial collagen content.
\end{abstract}

Clinical, physiological, and pathological studies of the pulmonary vascular tree have concentrated almost exclusively on the arterial side of the circulation. These studies have shown that adaptive changes - occurring over a wide range of pulmonary arterial pressures maintain the normal pulsatile flow in the lung capillaries even in the presence of severe pulmonary arterial hypertension (Lee and Du Bois, 1955; Bosman et al., 1964; Karatzas and Lee, 1970). This appears to depend on a reciprocal relationship between the resistance and compliance of the pulmonary arterial system that supplies the lung capillary bed. Thus, when pulmonary arterial resistance is increased, either physiologically or pathologically, there is an accompanying reduction in pulmonary arterial compliance so that the pulsatile inflow characteristics to the capillaries remain virtually unchanged (Reuben et al., 1970; 1971; Reuben, 1971).
The way in which the pulmonary venous system may act to protect lung capillary outflow from pressure events taking place in the left atrium is much less clear; for although the pulmonary veins possess no valves the profile of lung capillary blood flow is normally unaffected by left atrial pressure events (Gillespie et al., 1967; Karatzas and Lee, 1970). This implies that some part of the venous system can damp out retrograde pressure pulsations originating in the left atrium. This further implies that the pulmonary veins could be highly compliant.

Measurements of flow and pulse pressure patterns are relatively easy during cardiac catheterisation in man or experimental animals but a study of the static mechanical properties of the vessel wall requires necropsy material. Caro and Saffman (1965) have reported studies on the mechanical properties of the pulmonary arteries and veins in rabbits, as have Wolinsky and Glagov 
(1962) on the rabbit aorta. We can find no such studies of human pulmonary veins, although Harris, Heath, and Apostolopoulos (1965) have compared the extensibility of circumferential strips of human pulmonary artery and aorta obtained at necropsy. They showed that the compliance of human pulmonary arteries decreases with increasing age, that is, they become more resistant to stretching forces. They ascribed this change to an increase in the medial collagen content of the vessels. These investigations suggest that in animals and man the high tensile strength of collagen in the vessel wall is responsible for resisting excessive dilatation of the vessels at physiological internal pressures and above. The elastic laminae appear to function at lower internal pressures and act by distributing the pressure load evenly throughout the thickness and circumference of the wall.

We have now extended the observations of Harris and his colleagues (1965) to include mechanical, histological, and chemical studies of human pulmonary veins as well as arteries. We wished to test the compliance of pulmonary veins because of their likely importance in isolating pulmonary capillary blood flow from left atrial pressure events. We also studied the effect of age in an attempt to test the general hypothesis that an increasing medial collagen content was responsible for the increasing vessel wall stiffness with age.

\section{Material and methods}

Samples of pulmonary vessel wall were obtained at necropsy from the main pulmonary artery branches before the lobar divisions and from the pulmonary veins at the lung hilum, parenchyma, pericardial membranes, and loose areolar tissue being removed by gentle dissection. Any cases with a clinical history or post-mortem evidence of significant chronic cardiorespiratory disease (including mitral valve disease, congenital heart disease, chronic bronchitis and emphysema, recurrent pulmonary emboli, cardiomyopathy, or systemic hypertension) were excluded. The vessels were opened longitudinally, and circumferential strips, $2.5 \times 1.0 \mathrm{~cm}$, were cut from the wall with a special guillotine. The vessel strips were then weighed and placed in Krebs-Ringer solution until used for compliance measurements (usually within three to four hours of removal). These measurements, which are reported separately (Banks $e t$ al., 1977), were made using a tension balance method identical with that used by Harris et al. (1965). After compliance testing, the tissue samples, together with any unused vessel wall from the same site, were fixed in $10 \%$ neutral formol saline an $\overrightarrow{\vec{\phi}}$ stored in a cool, dark place until required.

HISTOLOGY

The strips of vessel wall were bisected longitudi 8 nally and one half was processed by standard histological methods and embedded in paraffin wax. In addition, the material from a few case? which had not been used in the tension balance् experiments was embedded for comparison. Seces tions were cut at $5 \mu \mathrm{m}$ thickness parallel to the long axis of the samples and stained by Verhoeff' method for elastin (black) and van Gieson' counterstain for collagen (red) and muscle (brown) All of the tissue processing and staining was perer formed by one experienced technician to main? tain uniformity of results so far as possible.

QUANTITATIVE EXAMINATION

The sections were checked microscopically to ensure that the media was intact, that the sections were cut at right angles to the surface, and for uniformity of staining. Quantitative examinatiob was then made to determine the proportions of elastin, muscle, and collagen in the wall by a poins counting method. A Leitz microprojector fitteक with a xenon lamp was used to project the section image on to a piece of card on which was draw a point counting grid based on a system of $2 \mathrm{cn}$ sided equilateral triangles. A magnification an $\Phi$ projection distance was selected to cover the fut thickness of the average arterial media and in cluded 77 counting points in each field. This wa\$ maintained throughout the study. Since the veif media is much thinner, a smaller diameter grio with a higher point density (108 points per field was used at the same magnification for theso vessels. In practice, it was found that counting $2 \vec{\Theta}$ fields (1540 points) for the arteries and 15 fields (1620 points) for the veins gave an adequate leved of accuracy. The counts were expressed as a per centage of the total possible points on the medias (as defined below) so that the histological results refer to this layer alone.

WALL THICKNESS

The thickness of the media alone and the full wall thickness were measured on all the point countef sections using an eyepiece micrometer calibrate against a graduated microscope reference slideo For these and the point counting measurement the media was defined as 'that part of the vesse wall between the external boundary of the intimg and the external limit of the muscular layer'. The full wall thickness was taken from the interna surface of the intima to the last of the elastif 
laminae externally rather than trying to guess at the limits of poorly defined and partially stripped adventitia. A minimum of 10 measurements were made at $1 \mathrm{~mm}$ intervals along the sections, taking consecutive steps unless artefactual damage was present, in which case the next clear area was taken. No attempt was made to correct for fixation-induced shrinkage artefact (which may be up to $20 \%$ of the original thickness) as all samples were processed in the same way and stored for approximately the same time before examination.

\section{CHEMICAL ANALYSIS}

The unembedded formalin-fixed halves of the vessel strips plus any remaining unused tissue from the same level of the wall were used for chemical estimation of their collagen content. These samples had the adventitial coat stripped in the same way and to the same extent as the stretched and/or histologically examined samples. To produce an adequate amount of material from a limited number of cases, samples were pooled in decade groups by age, as shown in Table 2 , and treated thereafter as a single representative sample of that group. The formalin fixed tissue was washed well and then dialysed against distilled water for a minimum of three days to remove traces of free formaldehyde. The samples were then freeze-dried and stored under vacuum with a silica gel desiccant until examined. The total hydroxyproline (OH-Pro) content in each batch was measured by the method of Bergman and Loxley (1970) using duplicate tests whenever possible. These figures were converted to total collagen by the appropriate factor and expressed as percentage collagen relative to the freeze-dried weight of the tissue samples.

\section{Results}

The full data and interpretation of the stressstrain measurements of vascular extensibility under load are reported elsewhere (Banks et al., 1977) but Fig. 1 summarises the effect of age on the extensibility of pulmonary artery and vein strips that was found. Briefly, there is a steady decrease in the extensibility of pulmonary artery strips with increasing age. The pulmonary veins, however, are much stiffer at all ages and show very little change with age. A biphasic shape to the arterial compliance curve is suggested in the younger cases, becoming more marked in the older age groups. This should be compared with the relatively flat venous curves at all ages. The steeply rising part of the curve probably represents stretching of the elastic laminae while the flatter

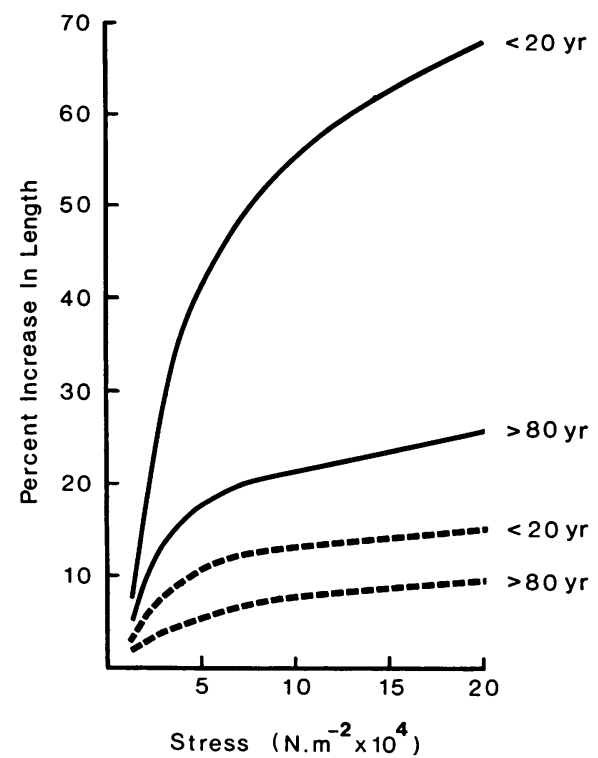

Fig. 1 Diagrammatic stress/strain curves of aging pulmonary arteries (- and pulmonary veins ( - . - . ) from data of Banks et al. (1977).

part indicates the greater involvement of inextensible collagen fibres. The results of the compliance studies were derived from measurements on 85 pulmonary veins and 62 pulmonary arteries out of a total of 112 pairs of specimens initially collected. Quantitative histological studies were made on 37 pulmonary vein samples and 42 arteries from approximately equal numbers of males and females aged $7-87$ years.

\section{QUANTITATIVE HISTOLOGY}

All of the vessels examined showed good preservation and staining with no recognisable autolytic damage and no difference between stretched and unstretched samples from the same case. The point counting system worked well and showed good agreement between observers on random duplicate counts. No clear pattern of results was apparent until the figures were plotted at the end of the study because the vessels had been taken in random order rather than in age sequence. At this stage, as shown in Fig. 2, it became obvious that our material did not show the increasing collagen content with age which Heath et al. (1959) had found in the pulmonary trunk and which Naeye and Dellinger (1971) had shown in the peripheral pulmonary vessels. In fact, the calculated regression lines for both arteries and veins showed a steady fall in collagen content of about $1 \%$ per decade with almost equal rates of 
fall for both vessels. The mean venous histological content was nearly twice that of arteries and maintained this ratio at all ages. Using Student's $t$

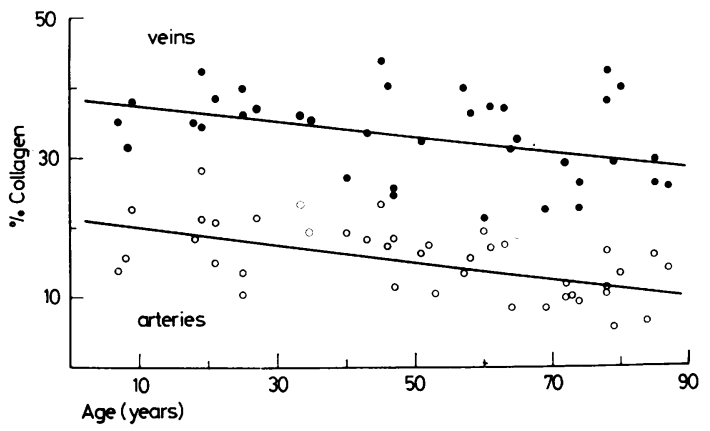

Fig. 2 Change in histological collagen content of normal human pulmonary vessel media with age. The slopes of the calculated regression lines (arteries: $y=21 \cdot 1-0 \cdot 114 x$; veins: $y=38.6-0.096 x$ ) are almost identical and the change is significant. test, the fall was significant when the under $3 \overrightarrow{\overrightarrow{\vec{A}}}$ years age group was compared with those oveo 70 years $(\mathrm{P}<0.002$ for arteries and $<0.05$ fo믄 veins). Although the correlation coefficients fop the regression lines were only $0.560(P<0.001)$ an $0.354(\mathrm{P}<0.05)$ for the arteries and veins respecto ively these were still significant for this relatively small number of cases.

Changes in the elastic and muscle proportions were less marked but there was an inverse relation ship between the two at all ages, and clear differ $\overrightarrow{\overrightarrow{2}}$ ences existed between arteries and veins. There was a tendency for the elastic content to fall witk age in the arteries (Table 1), as noted by Harris, et al. (1965), and also an increase in muscle conc్ tent in the older age groups, but no such tendencyo was seen in the veins.

The results of measuring the thickness of the vessel media are shown in Fig. 3. The actual thick ness of the vein media (discounting the effect of shrinkage) was very small, averaging about 6060

Table 1 Morphometric analysis of normal human pulmonary vessels giving range and mean values for each decade with wall thickness as defined in text

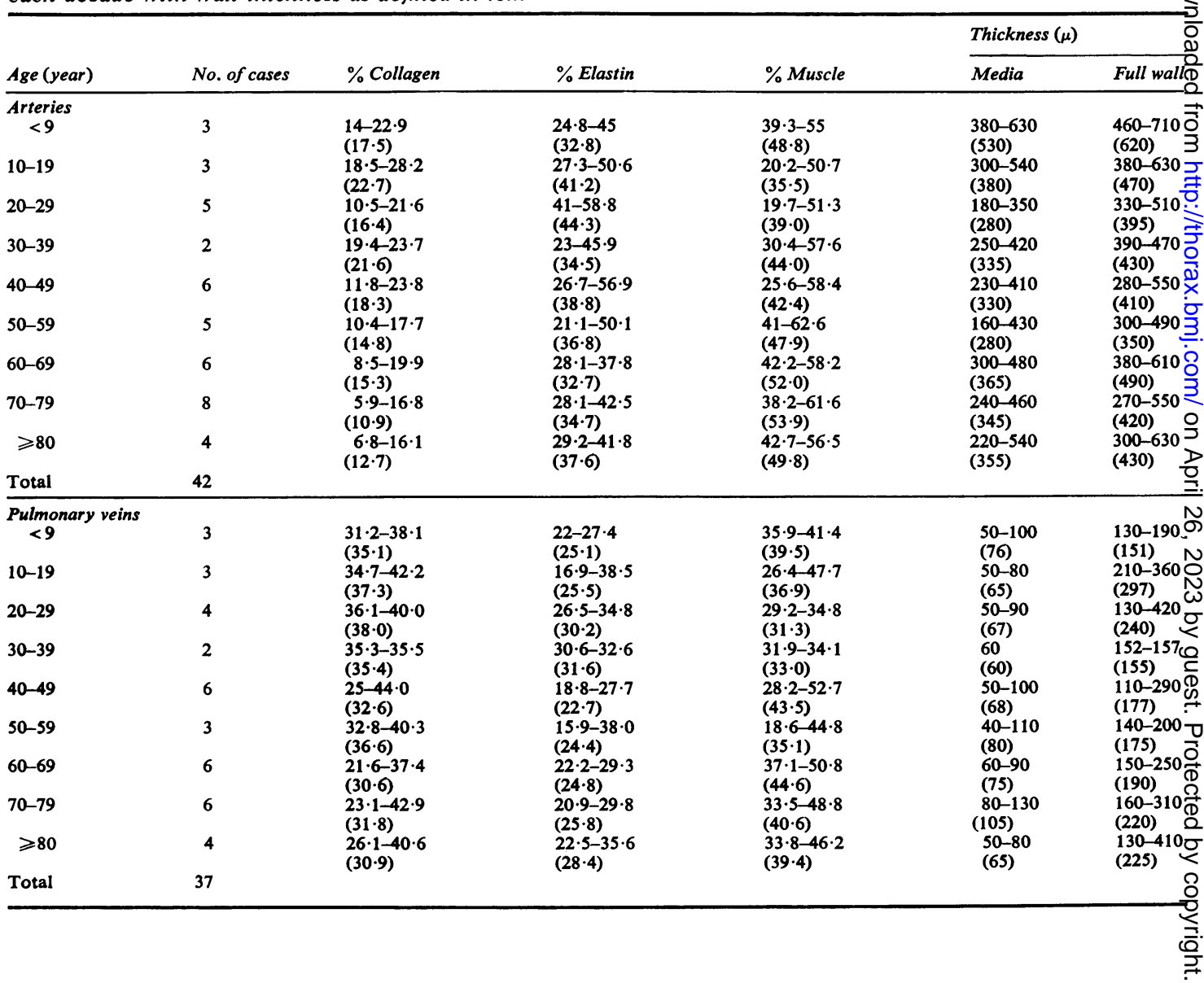




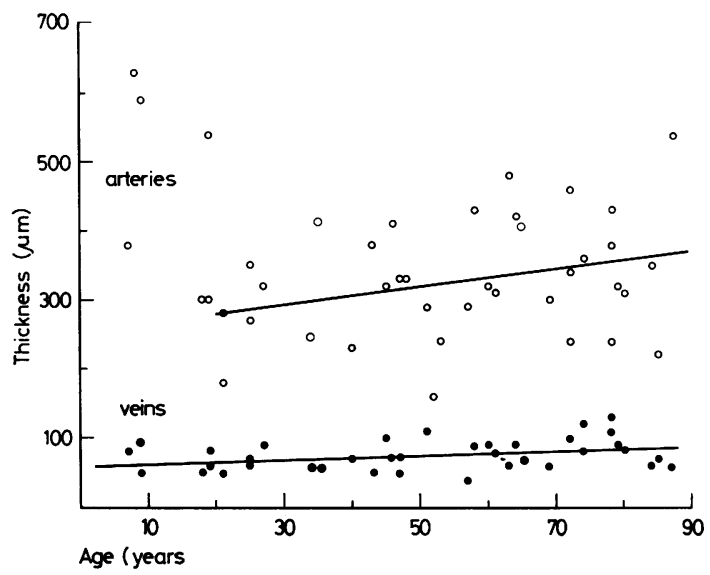

Fig. 3 Medial thickness in microns of normal pulmonary vessels. The increase in thickness with age is not significant ( $\mathrm{P}>0.10$ for arteries and veins).

$70 \mu \mathrm{m}$, and, although the regression line showed a slight upward slope, there was no significant change with age ( $P>0.10$ for both arteries and veins). The full wall thickness (about $200 \mu \mathrm{m}$ ) was much greater. This is because the elastic laminae gradually became further apart with increasing age. Collagen formed the greater part of the outer adventitial layer, which however was quite distinct from the media proper. Very few young arteries were examined and, as Fig. 3 shows, there was a very wide variation in thickness so that data from the first two decades have been excluded in calculating the regression line for arterial medial thickness and age. Even in the arteries the true media was very thin, averaging well under $500 \mu \mathrm{m}$. From the third to the ninth decades there was an apparent tendency to an increase in medial thickness but this was not statistically significant.

\section{CHEMICAL ESTIMATIONS}

These results are shown in Table 2 and plotted against age in Fig. 4. The values are derived from OH-Pro content expressed as percentage collagen. They include a small proportion derived from the medial elastin content plus a significant amount from the adventitial collagen remaining on the vessel wall. These two parts cannot be separately quantified on the results available but we estimate that they may contribute together not more than $10 \%$ of the collagen values derived from the total $\mathrm{OH}-$ Pro figures. It is thus particularly interesting that the chemical results showed the same ratio of collagen content between arteries and veins, a similar total decrease with age compared with the histological values, and a similar rate of decrease.
Table 2 Chemical collagen content of normal pulmonary vessels derived from hydroxyproline assay of formalin-fixed tissues expressed as percentage of freeze-dried weight of pooled tissue samples

\begin{tabular}{|c|c|c|c|c|c|}
\hline $\begin{array}{l}\text { Age } \\
\text { (yr) }\end{array}$ & $\begin{array}{l}\text { No. of } \\
\text { cases }\end{array}$ & $\begin{array}{l}\text { Dry } \\
\text { weight } \\
(m g)\end{array}$ & $\begin{array}{l}\text { Total } \\
\text { OH-Pro } \\
(\mathrm{mg})\end{array}$ & 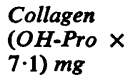 & $\%$ Collagen \\
\hline \multicolumn{6}{|c|}{ Pulmonary arteries } \\
\hline$<19$ & 7 & $105 \cdot 3$ & $5 \cdot 55$ & $39 \cdot 43$ & $37 \cdot 4$ \\
\hline $20-29$ & 6 & $49 \cdot 5$ & 3.03 & 21.48 & $43 \cdot 4$ \\
\hline 30-39 & 3 & $47 \cdot 7$ & $2 \cdot 36$ & $16 \cdot 76$ & $35 \cdot 1$ \\
\hline $40-49$ & 7 & 89.8 & $3 \cdot 61$ & $25 \cdot 60$ & $28 \cdot 5$ \\
\hline $50-59$ & 7 & $77 \cdot 6$ & $3 \cdot 13$ & $22 \cdot 22$ & $28 \cdot 6$ \\
\hline $60-69$ & 8 & 116.9 & $4 \cdot 29$ & 30.47 & $26 \cdot 1$ \\
\hline 70-79 & 10 & $110 \cdot 5$ & $4 \cdot 39$ & $31 \cdot 16$ & $28 \cdot 2$ \\
\hline$\geq 80$ & 5 & $67 \cdot 5$ & $2 \cdot 76$ & $19 \cdot 56$ & $29 \cdot 0$ \\
\hline \multicolumn{6}{|c|}{ Pulmonary veins } \\
\hline$<19$ & 7 & $72 \cdot 5$ & 6.96 & $49 \cdot 39$ & $68 \cdot 1$ \\
\hline $20-29$ & 6 & $30 \cdot 7$ & $2 \cdot 82$ & 19.99 & $65 \cdot 1$ \\
\hline $30-39$ & 3 & $22 \cdot 2$ & 2.06 & $14 \cdot 59$ & $65 \cdot 7$ \\
\hline $40-49$ & 7 & $52 \cdot \overline{5}$ & $4 \cdot 18$ & $29 \cdot 71$ & $56 \cdot 7$ \\
\hline $50-59$ & 7 & $41 \cdot 9$ & $2 \cdot 90$ & $20 \cdot 58$ & $49 \cdot 1$ \\
\hline $60-69$ & 7 & $74 \cdot 4$ & $6 \cdot 11$ & $43 \cdot 35$ & $58 \cdot 3$ \\
\hline $70-79$ & 10 & 50.5 & $3 \cdot 82$ & $27 \cdot 86$ & $55 \cdot 2$ \\
\hline$\geq 80$ & 5 & $37 \cdot 7$ & $2 \cdot 81$ & 19.97 & 53.0 \\
\hline
\end{tabular}

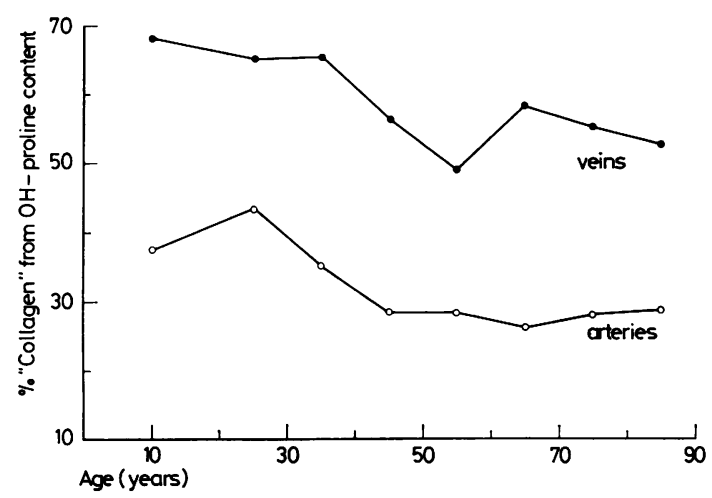

Fig. 4 Chemical collagen content of pulmonary vessel samples pooled in decade groups. The trend is similar to the reduction in histological collagen content.

The high collagen values in the pulmonary veins will include a larger proportion of collagen derived from the adventitia because the media was so thin. This was reflected in the much lower dry weight of the venous samples compared with the arteries for approximately the same total $\mathrm{OH}-\mathrm{Pro}$ content. Nevertheless, assuming a fairly constant residual adventitial layer, there was still a steady downward trend for medial collagen content in both pulmonary arteries and veins.

\section{QUALITATIVE HISTOLOGY}

To some extent the quantitative changes in the vessel wall components were accompanied by qualitative changes in their appearances, and these 
were most obvious in the elastic laminae. In the youngest patient (Fig. 5) the arterial elastin had lost the transitional type of configuration (that is, resembling the aortic media in appearance), and at 7 years of age it had parallel, nearly straight lamellae. There was relatively little collagen and a fairly high muscle content with an almost nonexistent intimal layer. With increasing age (Fig. 6), the elastic laminae showed a greater variation in thickness, and their arrangement became wavy and further apart. In the older age groups (Fig. 7), the intima became thickened and consisted of scanty fine collagen fibrils and even fewer elastic fibres in a loose mucopolysaccharide matrix.

In keeping with their lack of change in thickness and minor variation in muscle/elastin ratios, the

Figs. 5-8 Pulmonary vessel sections stained with $V$ erhoeff's elastic stain (black) and van Gieson counterstain. All photographed at $\times 192$ magnification. Histological composition of the media shown as percentage of area examined: $C=$ collagen, $E=$ elastin, $M=$ muscle, $T=$ thickness in microns. Arrowheads indicate limits of media as defined in text.

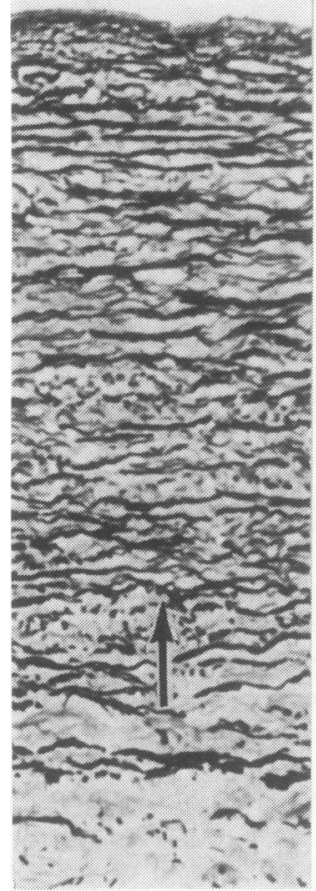

(a)

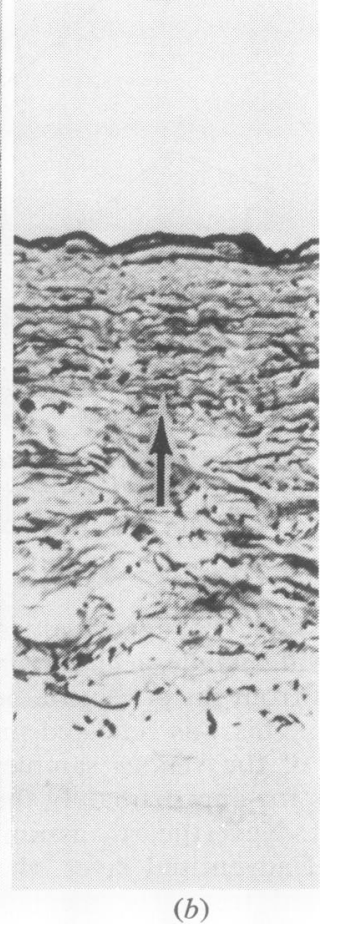

(b)
Fig. 5a Boy 7 yr. Pulmonary artery with thin straight elastic laminae and thin intimal layer (C $14 \%$, E $28.5 \%, M 55 \%, T 380 \mu$ ).

Fig. 5b Boy 7 yr. Pulmonary vein with well-defined internal elastic lamina and loose collagen/elastic adventitia $(C 36 \cdot 1 \%, E 22 \%, M 41 \cdot 1 \%, T 80 \mu)$.

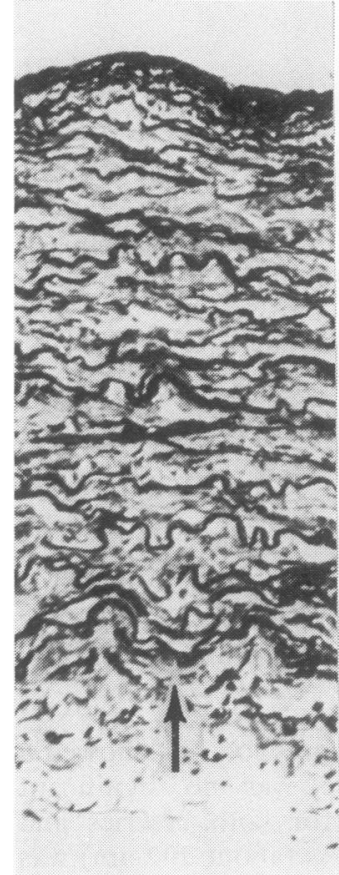

(a)

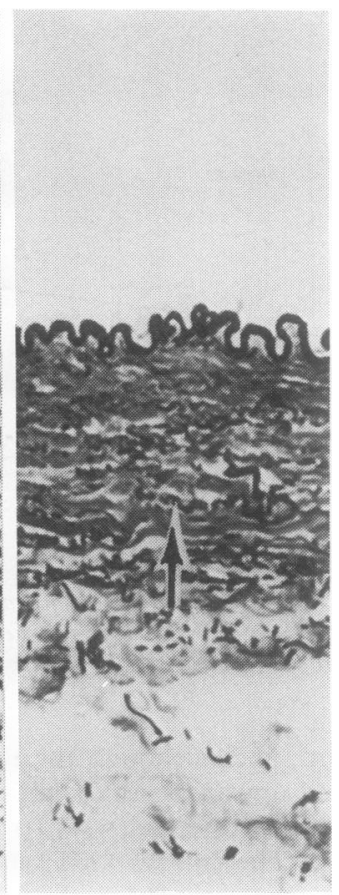

(b)
Fig. 6a Woman 47 yr. Pulmonary artery. Note condensation of subintimal elastic laminae and wavy outer layers (C 18.7\%, E 33.6\%, M 47.5\%, T $330 \mu$ ). Fig. $6 \mathrm{~b}$ Woman $47 y$ r. Pulmonary vein. Folded internal elastic lamina is probably artefact. Note plentiful irregular elastic fibrils in adventitia (C 25.5, E $27 \cdot 1 \%, M 45 \cdot 8 \%, T 70 \mu$ ).

veins showed no obvious variation with age, al- $\frac{x}{\partial}$ though this could be detected by quantitative ana- 3 . lysis. In a few sections, bundles of striated cardiac $ᄋ$ type muscle fibres were present in the residual 3 adventitial layer of the veins, possibly representing 음 the so-called 'sphincters' described by Nathan and Elaikim (1966).

It has been suggested that alteration in the properties of elastic tissue with increasing age is $ᄋ$ due to calcium deposition on the fibres (Lansing, $N$ 1959). To test this suggestion, two pairs of vessels $\underset{\omega}{N}$ from each age group were sectioned and stained by von Kossa's method for calcium salts. $\mathrm{No}_{6}$ deposits were seen at any age by this method al- $\frac{\mathbb{C}}{\mathbb{N}}$ though quite possibly minute traces could have $\stackrel{\mathcal{D}}{?}$ been present but not detectable by light micro- 0 scopy.

One abnormal case is reported for comparison $\stackrel{0}{\stackrel{\oplus}{\Phi}}$ (Fig. 8). A 35-year-old woman with severe rheumatic heart disease had mitral stenosis and in- $\frac{\varrho}{\sigma}$ competence with moderately severe pulmonary hypertension (PA pressure $70 / 36 \mathrm{mmHg}$, mean 


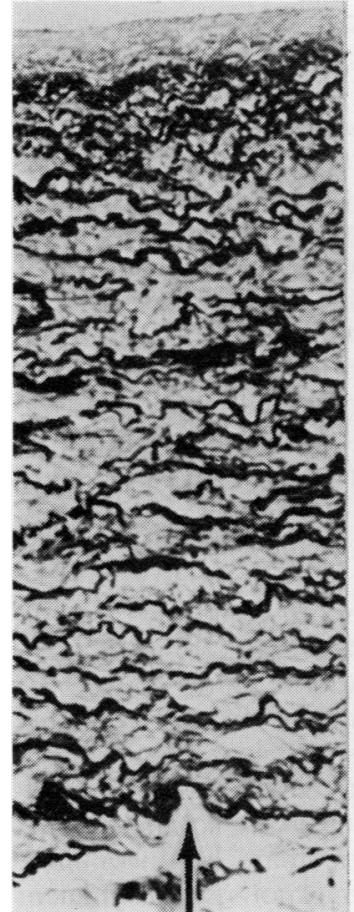

(a)

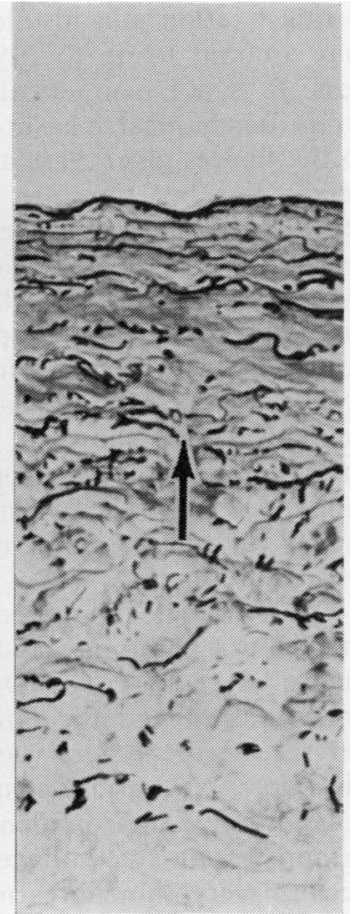

(b)
Fig. 7a Man 78 yr. Pulmonary artery. Intimal thickening and thickened irregular elastic laminae (C 16.8\%, E 42.5\%, M 38.2\%, T $430 \mu$ ).

Fig. 7b Man 78 yr. Pulmonary vein showing minimal change from younger vessels (C $38.9 \%$, E $20.9 \%$, $M 36 \%, T 130 \mu)$.

$55 \mathrm{mmHg}$ ). In this patient the pulmonary artery had an essentially normal composition (C $12 \cdot 1 \%$, E $47 \cdot 1 \%$, M $40 \cdot 7 \%$ ) with a normal adult elastic pattern. The vein, however, was considerably thickened (media $130 \mu$ ) with an increased smooth muscle content $(56 \cdot 7 \%)$, a low elastin $(15 \cdot 2 \%)$, and an average collagen content $(28 \cdot 1 \%)$.

\section{Discussion}

The structure and elastic properties of human pulmonary arteries and veins are strikingly different and reflect differences in the functions of these vessels rather than just their mean intravascular pressures. The pulmonary artery is required to show a limited degree of distensibility to accommodate a bolus of blood from the right ventricle in systole and subsequently to add further impetus to onward flow from the elastic recoil of its wall. The circumferential arrangement of a network of elastic laminae serves this function while an interstitial and adventitial feltwork of virtually

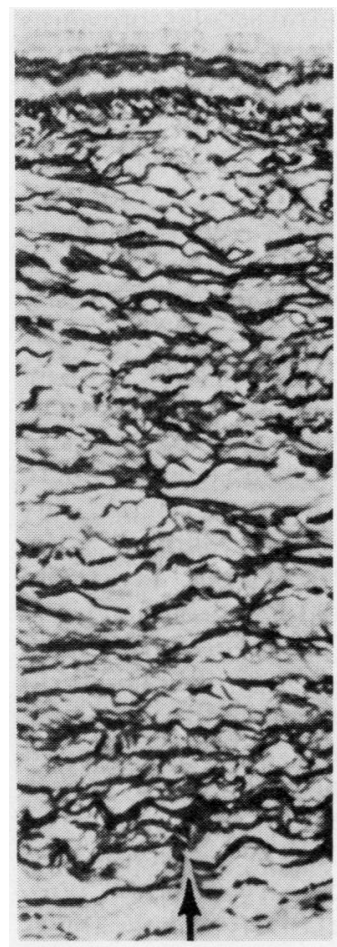

(a)

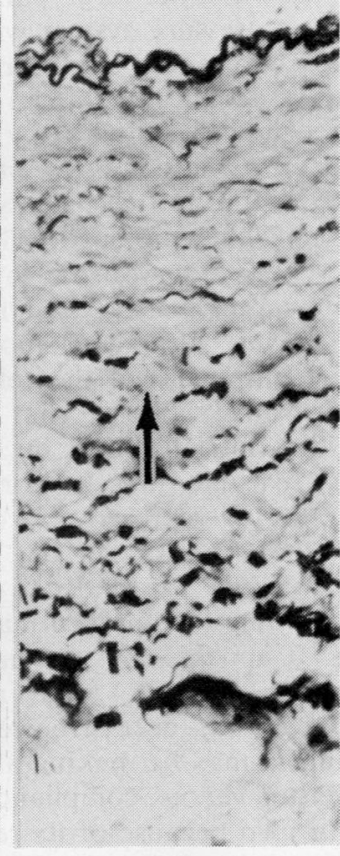

(b)
Fig. 8a Woman 35 yr with severe rheumatic heart disease and pulmonary hypertension. Pulmonary artery shows intimal and medial thickening with condensation of elastic laminae (C $13 \cdot 2 \%, E 38.2 \%, M 48.6 \%$, $T 400 \mu$ ).

Fig. 8b Woman 35 yr (same case as in Fig. 8a). Thickened pulmonary vein with subintimal elastic condensation and muscular hypertrophy of media (C $30 \cdot 1 \%$, E $17 \%, M 52.6 \%, T 130 \mu$ ).

inextensible collagen fibres prevents wasteful overdistension. The fine structure of pulmonary arteries shows a similar pattern to that of systemic elastic arteries (Pease and Paule, 1960), the main differences being quantitative only. Electron microscopy confirms the continuity between the elastic laminae and smooth muscle cells, the latter forming diagonal bridges between the laminae. Both are enclosed in a common basement membrane and may be expected to act in unison as one part of a two-phase system. The collagen fibres forming the other part of the system are quite separate and have no physical union with the other components. This two-phase structural arrangement is usually quoted as the explanation for the shape of the arterial compliance curve (Fig. 1). The steeply rising part of the curve corresponds to the physiological pressure range while 
the flatter part represents intravascular pressures well over the normal upper limit.

In contrast, the large pulmonary veins are part of a low pressure system which fills to a circular cross section profile in atrial systole but which collapses during rapid atrial filling (Bertram et al., 1977). They show relatively large volume changes within the physiological pressure range but, as shown in Fig. 1, are virtually non-distensible structures at higher pressures. The veins effectively act as a venous reservoir and by rapid filling in atrial systole largely absorb the retrograde atrial pressure wave. Although smooth muscle forms a major component of the vein wall structure there is no positive evidence that it plays an active part in aiding atrial filling in health. Similarly, the scanty cardiac muscle fibres in the adventitia, thought to represent a cardiac sphincter by Nathan and Elaikim (1966), are highly unlikely on morphological grounds to contribute much to atrial filling or prevention of reflux into the veins. It appears that the medial collagen content plus the adventitial coat are the main factors in preventing excessive venous dilatation. The adventitia may be making a larger contribution to the flatter venous compliance curves both in vitro and in vivo because of its relatively greater thickness on the veins compared with the medial thickness of veins or arteries.

Our experiments confirm those of Harris et al. (1965), showing that the pulmonary arterial wall becomes stiffer with increasing age. There is also an increase in venous wall stiffness but to a much smaller degree. We have shown that this increase in stiffness is accompanied by a steady decrease in medial collagen content in arteries and veins on quantitative histological and biochemical grounds. This is in direct contrast to the non-quantitative morphological observations of Heath et al. (1959), who reported an increase in the collagen content of the pulmonary trunk with increasing age. Chemical analysis by Farrar, Blomfield, and Reye (1965) showed an increase in collagen in the main pulmonary artery but a decrease in its main branches of a similar order to our results. In parallel with the relatively minor functional changes, we found that the pulmonary veins showed little morphological change with age alone. A decrease in their collagen content was evident only on morphometry and chemical analysis.

Measurements of medial thickness showed no significant change with age in either the pulmonary arteries or veins, although we confirmed a slight tendency to an increase in the arterial media, as found by Harris et al. (1965). Our figures do not take account of possible shrinkage during fixation and histological processing, which may amount to up to $20 \%$ of the original thickness. It is not a practical proposition to attempt direct mechanical measurement of wall thickness so the figures given here are recognised to be artificially low but they are comparable to those of previous studies. However, by comparison with systemic arteries, the media of the main pulmonary vessels are very thin in keeping with their low pressure function. In pulmonary hypertension, both venous and arterial media become thickened, largely as a result of an increased smooth muscle content. This is shown in Fig. 8 and suggests that smooth muscle hypertrophy in the main pulmonary veins may be an important response in mitral valve disease when it may actively help in left atrial filling.

The histological point counting method of quantitation was based on standard morphometric techniques as reviewed by Dunnill (1968). With the grid we used and the number of points counted per section the system was sufficiently accurate to pick up a $2 \%$ variation in the smallest wall component with $95 \%$ confidence. Even so, histological demonstration of collagen may be incomplete because very thin fibres may not retain sufficient $\stackrel{0}{\circ}$ stain to show at the magnification used. Such $\stackrel{\unrhd}{\unrhd}$ fibres would, of course, be included in biochemical $\overrightarrow{\vec{A}}$ estimates and account for part of the higher values derived from the $\mathrm{OH}$-Pro measurements.

Collagen is relatively resistant to post-mortem autolysis and should not be greatly affected by the variable death-to-necropsy interval in these cases. 음 Current theories of the chemical nature of for- $\underset{x}{x}$ malin fixation (Baker, 1960) would predict no $\frac{\dot{0}}{\partial}$ reaction with hydroxyprolyl residues which do not have side chain amino groups available for $\delta$ reaction with the aldehyde. It was these considerations which prompted us to try chemical measure-을 ment of pulmonary vessel collagen using formalin-fixed specimens as a control for the un-음 expected findings on quantitative histology. $A_{\tilde{N}}$ similar approach was successfully employed by 0 Montfort and Pérez-Tamayo (1962) to estimate $N$ the collagen content of fresh and formalin-fixed N myocardium.

We have found that the marked reduction in static wall compliance, which takes place with age, is not due to an increase in the medial collagen content as commonly suggested; indeed, we have shown that there is a distinct fall with age in $\overrightarrow{0}$ this component. An alternative explanation is therefore required.

It is known that intermolecular collagen crosslinks increase with age, and this increases the tensile strength of individual fibres. It is not pos- - 
sible to assess the contribution which this process makes towards the mechanical changes seen in Fig. 1 but we feel that it is unlikely to be solely responsible since the stress at which these changes become noticeable is well below the theoretical breaking point of collagen fibres. It is more likely that analogous intermolecular reactions, which are known to occur in elastin, restrict its ability to deform under small loads. If, as Roach and Burton (1957) suggested, the first rapid extension phase of the stress/strain curve mainly represents stretching of the elastic tissue, consideration of Fig. 1 and the data of Harris et al. (1965) and Banks et al. (1977) shows that the main area of change with age is in the elastic phase of the curve. In this case, the reduction of wall compliance with age, coupled with a reducing medial collagen content, strongly suggests that it is the elastic component approaching its elastic limit at lower loads which produces the flatter curves in the older age groups. Although age-related changes at the molecular level may contribute to these mechanical alterations, it remains a possibility that the morphologically visible changes in the arrangement of these fibres at the lamellar level may be more relevant (Figs. 5-7).

The reason for the decrease in collagen content of the pulmonary vessels and the way in which it occurs must remain speculative at this stage. Collagen is not inert and undergoes a slow but definite metabolic turnover. Most reports suggest that in non-diseased parenchymal organs there is a constant parenchyma: connective tissue ratio with age and an increase in total collagen in tissues such as skin and aorta. Again the mechanisms are not clear but depend partly on such factors as local blood flow and the effect of sex and growth hormones. The steady rate of decrease in histological collagen content seen in our vessels is similar to the decrease in bone mass with increasing age, though at a much slower rate. The almost identical rates of collagen decrease in arteries and veins suggests that this is a genuine reduction, which may be a physiological response to increased load-bearing by the stiffer elastic tissue present in the media of both arteries and veins with increasing age.

Post-mortem compliance tests take no account of the possible active part played by smooth muscle cells in maintaining wall tension or producing active changes. Autolysis leaves these cells as viscous fillers between the elastic laminae, making a small but not directly measurable contribution to the compliance results. There is clearly a need for experiments on viable vessel walls to take account of smooth muscle activity.
In conclusion, the decrease in pulmonary artery compliance which we found with increasing age in the absence of pulmonary arterial hypertension appears to be the result of structural changes in its medial elastic tissue. Similar changes occurred in the pulmonary veins and, in both, quantitative analysis showed a steady fall in the medial collagen content with increasing age. We suggest that increasing intermolecular linkage of elastin as well as collagen may be responsible for the mechanical changes taking place in the pulmonary vessels with age. We have also found that at no age do the pulmonary veins behave as compliant structures. We must therefore postulate that their protective role in isolating pulmonary capillaries from left atrial pressure changes depends on a capacitance effect due to their collapsible nature rather than distensibility. This suggestion has now been tested and is reported briefly elsewhere (Bertram et al., 1977).

We thank Mr. A. Chaplin, FIMLS, for careful preparation of the histological sections, Mrs. S. Hawker for the hydroxyproline estimations, and Dr. T. M. Parry for the photomicrographs.

\section{References}

Baker, J. R. (1960). Principles of Biological Microtechnique. Methuen, London.

Banks, J., Booth, F. V. McL., MacKay, E. H., Rajagopalan, B., and Lee, G. de J. (1977). The physical behaviour of human pulmonary arteries and veins. Clinical Science. (In press).

Bergman, I., and Loxley, R. (1970). The determination of hydroxyproline in urine hydrolysates. Clinica Chimica Acta, 27, 347-349.

Bertram, C. D., Lee, G. de J., Rajagopalan, B., and Stallard, T. (1977). Measurement of the dimensions of the extraparenchymal pulmonary veins in the dog. Journal of Physiology, 272, 101-102.

Bosman, A. R., Honour, A. J., Lee, G. de J., Marshall, R., and Stott, F. D. (1964). A method for measuring instantaneous pulmonary capillary blood flow and right ventricular stroke volume in man. Clinical Science, 26, 247-260.

Caro, C. G., and Saffman, P. G. (1965). Extensibility of blood vessels in isolated rabbit lungs. Journal of Physiology, 178, 193-206.

Dunnill, M. S. (1968). Quantitative methods in histology. In: Recent Advances in Clinical Pathology, Series V, edited by S. C. Dyke, pp. 401-416. Churchill, London.

Farrar, J. F., Blomfield, J., and Reye, R. D. K. (1965). The structure and composition of the maturing pulmonary circulation. Journal of Pathology and Bacteriology, 90, 83-96.

Gillespie, W. J., Greene, D. G., Karatzas, N. B., and Lee, G. de J. (1967). Effect of atrial systole on right 
ventricular stroke output in complete heart block. British Medical Journal, 1, 75-79.

Harris, P., Heath, D., and Apostolopoulos, A. (1965). Extensibility of the human pulmonary trunk. British Heart Journal, 27, 651-659.

Heath, D., Wood, E. H., Dushane, J. W., and Edwards, J. E. (1959). The structure of the pulmonary trunk at different ages and in cases of pulmonary hypertension and pulmonary stenosis. Journal of Pathology, and Bacteriology, 77, 443-456.

Karatzas, N. B., and Lee, G. de J. (1970). Instantaneous lung capillary blood flow in patients with heart disease. Cardiovascular Research, 4, 265-273.

Lansing, A. I. (1959). The Arterial Wall. Baillière, Tindall and Cox, London.

Lee, G. de J. (1971). Regulation of the pulmonary circulation. British Heart Journal, 33, Supplement, 15-26.

Lee, G. de J., and Du Bois, A. B. (1955). Pulmonary capillary blood flow in man. Journal of Clinical Investigation, 34, 1380-1389.

Montfort, I., and Pérez-Tamayo, R. (1962). The muscle-collagen ratio in normal and hypertrophic human hearts. Laboratory Investigation, 11, 463470.

Naeye, R. L., and Dellinger, W. S. (1971). Pulmonary arterial changes with age and smoking. Archives of Pathology, 92, 284-288.

Nathan, H., and Eliakim, M. (1966). The junction between the left atrium and the pulmonary veins.
An anatomic study of human hearts. Circulation, 34, 412-422.

Pease, D. C., and Paule, W. J. (1960). Electron microscopy of elastic arteries; the thoracic aorta of the rat. Journal of Ultrastructural Research, 3, 469483.

Reuben, S. R. (1971). Compliance of the human pul- क monary arterial system in disease. Circulation $\vec{\circ}$ Research, 29, 40-50.

Reuben, S. R., Gersh, B. J., Swadling, J. P., and Lee, G. de J. (1970). Measurement of pulmonary artery distensibility in the dog. Cardiovascular Research, $\vec{x}$ 4, 473-481.

Reuben, S. R., Swadling, J. P., Gersh, B. J., and Lee, $\dot{\omega}$ G. de J. (1971). Impedance and transmission pro- $\omega$ perties of the pulmonary arterial system. Cardio- $\mathcal{W}_{\mathrm{T}}$ vascular Research, 5, 1-9.

Roach, M. R., and Burton, A. C. (1957). The reason for the shape of the distensibility curves of arteries. Canadian Journal of Biochemistry, 35, 681-690.

Wolinsky, H., and Glagov, S. (1964). Structural basis for the static mechanical properties of the aorta $\overrightarrow{0}$ media. Circulation Research, 14, 400-413.

Requests for reprints to: Dr. E. H. MacKay, Department of Pathology, Leicester General Hospital, $\mathbb{D}$ Leicester LE1 5WW. 